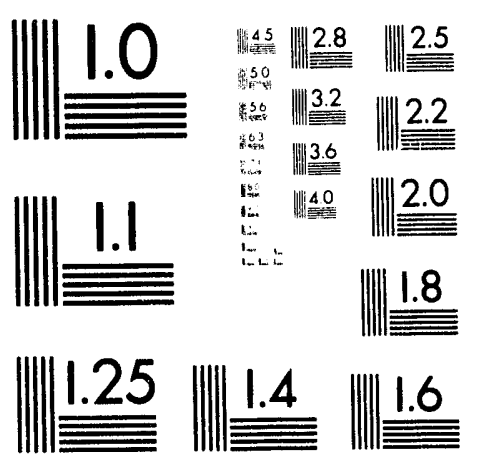



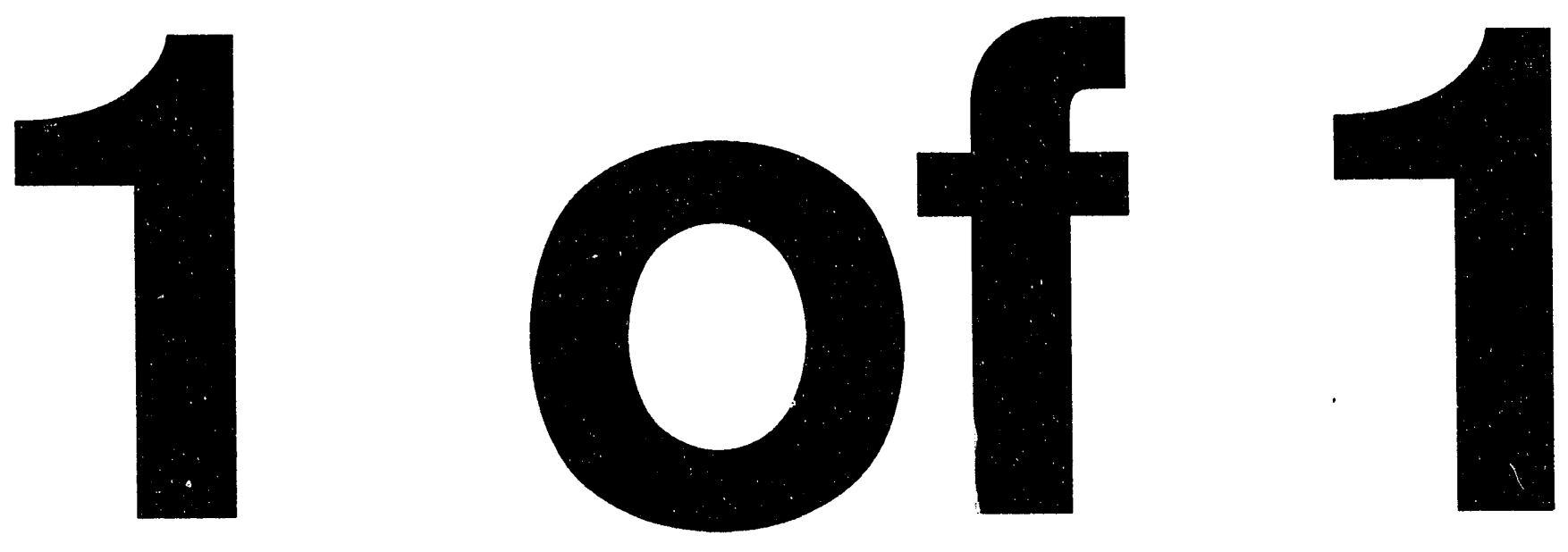
SAND93-0051

Distribution

Unlimited Release

Category UC-706

Printed July 1993

\title{
A Laboratory Evaluation of Color Video Monitors
}

\author{
Preston L. Terry \\ Imaging Technologies and Systems Department \\ Sandia National Laboratories \\ Albuquerque, New Mexico, 87185
}

\begin{abstract}
Sandia National Laboratories has considerable experience with monochrome video monitors used in alarm assessment video systems. Most of these systems, used for perimeter protection, were designed to classify rather than to identify intruders. There is a growing interest in the identification function of security video systems for both access control and insider protection. Because color video technology is rapidly changing and because color information is useful for identification purposes, Sandia National Laboratories established a program to evaluate the newest relevant color video equipment. This report documents the evaluation of an integral component, color monitors. It briefly discusses a critical parameter, dynamic range, details test procedures, and evaluates the results.
\end{abstract}

This work was supported by the US Department of Energy under Contract DE-AC04-DP00789.

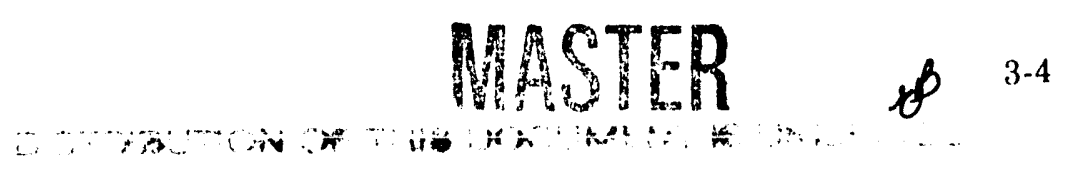




\section{CONTENTS}

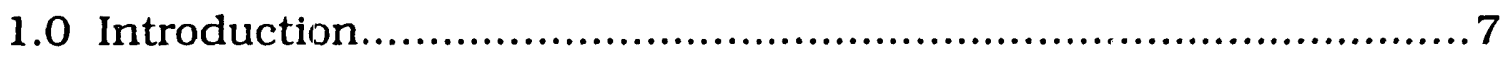

2.0 Monitor Dynamic Range Evaluation ........................................

2.1 Test Setup ............................................................

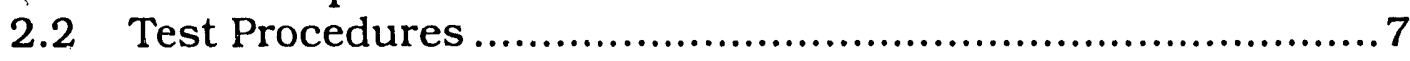

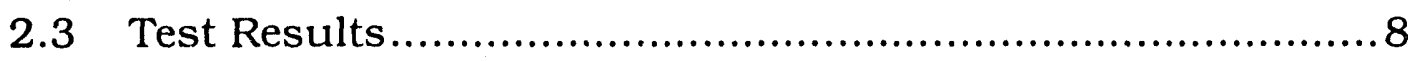

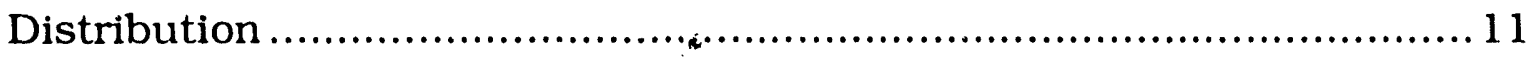

Table

1 Dynamic Range Test Results Table ........................................ 9 


\section{A Laboratory Evaluation of Color Video Monitors}

\subsection{INTRODUCTION}

Color monitors translate the electrical signal they receives from cameras into a visual image; therefore, performance is critical when used in a CCTV system. An evaluation was conducted on seven monitors to determine their dynamic range and resolution performance. Monitor selection criterion were:

- different screen sizes

- different screen sizes and different manufacturers

- different manufacturers.

\subsection{MONITOR DYNAMIC RANGE EVALUATION}

The dynamic range of a monitor is defined as the range of highlight luminance level that can be achieved while maintaining the complete ten step gray scale response and without any appreciative degradation in resolution. The dynamic range is important in determining the ambient light level in which the monitor can operate. Brighter screens are necessary when viewed in an environment with higher ambient light levels. The following paragraphs describe the test setup and procedures used for evaluating the monitors' dynamic range.

\subsection{Test Setup}

A camera was set up to view a backlit EIA resolution chart. The video output of the camera was attached to the input of a waveform monitor. The output of the waveform monitor was connected to the input of a video monitor.

\subsection{Test Procedures}

Initially, while viewing the resolution chart displayed by the monitor, the contrast and brightness controls were set to maximum. At this setting a horizontal resolution reading was taken, and the number of gray scale 
steps reproduced by the monitor were counted. The results were tabulated along with the measured luminance intensity of the monitor. The luminance intensity of the monitors were measured in the top and bottom inner portion of the central circle of the EIA chart. One measurement was taken at the top and another at the bottom quadrant of the circle in order to get an indication of the uniformity of the intensity.

If ten steps of gray scale were discerned and if the measured resolution was equal to or better than the manufacturer specification, then no other measurements were taken. If the monitor failed to reproduce either ten gray steps or the specified resolution, then the contrast and brightness levels were adjusted to the point where the monitor best performed, and the results were tabulated. The table below gives the results of the evaluation.

\subsection{Test Results}

To check the resolution of color monitors the camera used should provide greater resolution. However, at the time of this evaluation there were no known color video cameras made for security applications that met that requirement. The highest resolution for the cameras was 480 horizontal television lines compared to 600 for the monitors, therefore, some of the monitors were not tested for their maximum performance. Keeping that fact in mind all of the cameras performed as expected except the Barco AVM-22H and the Panasonic CT-1400MG. Their resolution at full brightness/contrast was considerable lower than the published specifications. Also, it should be noted that the Panasonic monitor only produced eight of the ten gray scale steps.

All the monitors except the JVC VMR-190SU displayed relative luminance intensity uniformity over the entire screen. 


\section{Dynamic Range Test Results Table}

\begin{tabular}{|c|c|c|c|c|c|c|}
\hline $\begin{array}{l}\text { MANUFACTURER } \\
\text { AND } \\
\text { MODEL NUMBER }\end{array}$ & Screen Size & $\begin{array}{l}\text { Horizontal } \\
\text { Resolution } \\
\text { Specification }\end{array}$ & $\begin{array}{l}\text { Horizontal } \\
\text { Resolution } \\
\text { Test } \\
\text { Results }\end{array}$ & $\begin{array}{l}\text { Scree } \\
\text { Meast } \\
\text { Foot } \\
\text { Top }\end{array}$ & $\begin{array}{l}\text { inance } \\
\text { tt in } \\
\text { rts. } \\
\text { Bottom }\end{array}$ & $\begin{array}{l}\text { Number of } \\
\text { Gray Scale } \\
\text { Steps Displayed }\end{array}$ \\
\hline $\begin{array}{l}\text { JVC } \\
\text { VMR-190SU }\end{array}$ & 19 inches & 480 TVL & 480 TVL & 57 & 34 & 10 \\
\hline $\begin{array}{l}\text { SONY } \\
\text { PVM- 1344Q }\end{array}$ & 13 inches & $600 \mathrm{TVL}$ & *480 TVL & 69 & 70 & 10 \\
\hline $\begin{array}{l}\text { JAVELIN } \\
\text { CVM-9 }\end{array}$ & 9 inches & $330 \mathrm{TVL}$ & $320 \mathrm{TVL}$ & 83 & 80 & 10 \\
\hline $\begin{array}{l}\text { BARCO } \\
\text { AVM-22IH }\end{array}$ & 10 inches & $500 \mathrm{TVL}$ & $<200$ TVL & 81 & 85 & 10 \\
\hline $\begin{array}{l}\text { BARCO } \\
\text { AVM-22IH }\end{array}$ & 10 inches & 500 TVL & $450 \mathrm{TVL}$ & 29 & 29 & 10 \\
\hline $\begin{array}{l}\text { PANASONIC } \\
\text { BTM-1310Y }\end{array}$ & 13 inches & $550 \mathrm{TVL}$ & *470 TVL & 49 & 43 & 10 \\
\hline $\begin{array}{l}\text { PANASONIC } \\
\text { CT-1400MG }\end{array}$ & 14 inches & $370 \mathrm{TVL}$ & $280 \mathrm{TVL}$ & 57 & 54 & 8 \\
\hline $\begin{array}{l}\text { PANASONIC } \\
\text { CT-1400MG }\end{array}$ & 14 inches & $370 \mathrm{TVL}$ & $350 \mathrm{TVL}$ & 48 & 46 & 10 \\
\hline $\begin{array}{l}\text { SONY } \\
\text { PVM-8221 }\end{array}$ & 8 inches & $400 \mathrm{TVL}$ & $410 \mathrm{TVL}$ & 48 & 50 & 10 \\
\hline
\end{tabular}

* Denotes resolution was limited by the camera's resolution of 480 horizontal television lines. 


\section{Distribution:}

1 Richard A. Clayton

Acting A: st. Secy.Defense Programs

U.S. DOE DP-1

Washington, DC 20585

1 RAdm. W.G. Ellis, USN

Dep. Asst. Secy. for Military

Application

U.S. DOE

DP-20

Washington, DC 20585

1 W.L. Barker, Acting Director

Office of Security Affairs

SA-1

U.S. DOE

Washington, DC 20585

1 A. Bryan Siebert, Director Office of Classification

U.S. DOE

SA-20

Washington, DC 20585

1 E.J. McCallum, Director

Office of Safeguards and Security

U.S. DOE

SA-10

Washington, DC 20585

1 O.B. Johnson, Deputy Director

Office of Safeguards and Security

U.S. DOE

SA-10

Washington, DC 20585

1 A. Barry Dalinsky, Chief Personnel Security, Procedures/ Analysis Branch

Office of Safeguards and Security

U.S. DOE

SA-122

Washington, DC 20585

1 William J. Desmond, Chief

Physical Security Branch

Policy, Standards \& Analysis Division

Office of Safeguards and Security

U.S. DOE

SA-121

Washington, DC 20585 
1 Larry D. Wilcher, Chief

Tech \& Operations Security Branch

Office of Safeguards and Security

U.S. DOE

SA-123

Washington, DC 20585

1 Brook Todd, Chief

Assessment and Intergration Branch

Office of Safeguards and Security

U.S. DOE

SA-131

Washington, DC 20585

1 Ernest E. Wagner, Chief

Weapons Safeguards and Security

Operations Branch

Office of Safeguards and Security

U.S. DOE

SA-132

Washington, DC 20585

1 William Hensley, Director

Processing and Reactors Facilities

S\&S Division

U.S. DOE

DP-681

Washington, DC 20585

1 A.J. Heysel, Chief

Production/Energy Safeguards/

Security Operations Branch

Office of Safeguards and Security

U.S. DOE

SA-133

Washington, DC 20585

1 Carl A. Pocratsky

Planning \& Tech Dvlp Branch

Office of Safeguards \& Security

U.S. DOE

SA-134

Washington, DC 20585

1 Dan Smith, Chief

Planning and Technology

Development Branch

Office of Safeguards and Security

U.S. DOE

SA-134

Washington, DC 20585 
1 Marshall O. Combs, Director

HQ Operations Division

Office of Safeguards and Security

U.S. DOE

SA-14

Washington, DC 20585

1 Charles Coker, Chief

HQ Physical Protection Branch

Office of Safeguards and Security

U.S. DOE

SA-141

Washington, DC 20585

1 Robert J. Caldwell, Chief

HQ Technical \& Information Security Branch

Office of Safeguards and Security

U.S. DOE

SA-142

Washington, DC 20585

1 Kenneth E. Sanders, Director

International Safeguards Division

U.S. DOE

DP-5.22

Washington, DC 20585

1 Robert J. Walsh, Depurty Director

Office of Security Affairs

U.S. DOE

SA-1

Washington, DC 20585

1 Philip G. Sewell,

Dep. Asst. Secy. for Uranium

Enrichment

U.S. DOE

NE-30

Washington, DC 20585

1 E.C. Brolin, Dep. Asst. Secy. for Civilian

Reactor Development

U.S. DOE

NE-40

Washington, DC 20585

1 Stephen J. Lanes

Dep. Asst. Secy. for Space and

Defense Systems

U.S. DOE

NE-50

Washington, DC 20585 
1 David E. Bailey, Director Fuels and Reprocessing Division U.S. DOE/ NE-471

Washington, DC 20585

1 Adm. Bruce Demars, USN

Dep. Asst. Secy. for Naval Reactors

U.S. DOE/NE-60

Washington, DC 20585

1 John W. Bartlett, Director Office of Civilian Radioactive

Waste Management

U.S. DOE/RW-1

Washington, DC 20585

1 Kenneth H. Besecker, Director Safeguards and Security Division U.S. DOE/Savannah River P.O. BOX A

Aiken, SC 29802

1 Don Jewell, Asst. Director Central Training Academy U.S. DOE/Albuquerque P.O. BOX 18041

Albuquerque, NM 87185

1 D.A. Gurule, Director Security \& Nuclear Safeguards Division U.S. DOE/Albuquerque P.O. BOX 5400 Albuquerque, NM 87115

1 L.J. Hofferth, Chief Physical Security Branch Security \& Nucl Safeguards Division U.S. DOE/Albuquerque P.O. BOX 5400

Albuquerque, NM 87115

1 A.R. Chernoff U.S. DOE/Albuquerque P.O. BOX 5400 Albuquerque, NM 87115

1 J.L. Loftis, Chief Operations Branch Security and Nuclear Safeguards Division U.S. DOE/Albuquerque P.O. BOX 5400 Albuquerque, NM 87115 
1 Joe Lewis, Asst. Director Central Training Academy

U.S. DOE/Albuquerque

P.O. BOX 18041

Albuquerque, NM 87185

1 R.E. Sabre, Director Transportation Safeguards Division U.S. DOE/Albuquerque

P.O.BOX 5400

Albuquerque, NM 87115

1 Roger D. Rollins, Director

Quality \& Materials Assurance Division

U.S. DOE/SR

P.O. BOX A

Aiken, SC 29802

1 Dennis Vernon, Chief

Planning and Liaison Branch

U.S. DOE/SR

P.O. BOX A

Aiken, SC 29802

1 John M. Sameuls, Manager

Safeguards and Security

Westinghouse Savannah River Co.

P.O. BOX 616

Aiken, SC 29802

1 Howard, Gnann, Chief

Safeguards Engineering \& Project Branch

U.S. DOE/SR

P.O. BOX A

Aiken, SC 29802

1 Richard Phillips, Chief

Safeguards \& Security Mgmt Branch

U.S. Doe/Amarillo

P.O. BOX 30030

Amarillo, TX 79120

1 Carleton, Bingham

U.S. DOE/Ch

New Brunswick Laboratory

9700 South Cass Avenue

Argonne, IL 60439

1 H.W. Kelly, Director

Safeguards and Security Division

U.S. DOE/Ch

9800 South Cass Avenue

Argonne, IL 60439 
1 Thomas Gradle, Chief

Security Operations Branch

U.S. DOE/Ch

9800 South Cass Avenue

Argonne, IL 60439

1 Joan Christopher, Security Officer

Western Area Power Administration

U.S. DOE/RF

P.O. BOX 928

Golden, CO 80401

1 R.J. Levernier, Director

Safeguards and Security Branch

U.S. DOE/RF

P.O. BOX 928

Golden, CO 80402-0928

1 H.R. Martin, Acting Director

Safeguards and Security Division

U.S. DOE/Ida'u,

785 DOE Place

Idaho Falls, ID 83402

1 Administrator

Alaska Power Administration

U.S. DOE

P.O. BOX 50

Juneau, AK 99802

1 Roger Teska, Chief

Security Section

U.S. DOE/KC

P.O. BOX 202

Kansas CIty, MO 64141

1 E.W. Adams, Director

Safeguards and Security Division

U.S. DOE/NV

P.O. BOX 98518

Las Vegas, NV 89193-8518

1 Jean McGarry, Chief

Safeguards and Security Branch

U.S. DOE/NV

P.O. BOX 435

Mercury, NV 89023

1 Donald J. Ornick, Director

Security Division

U.S. DOE/OR

900 Commerce Road East

New Orleans, LA 70123 
1 Jack Blasy, Director

Safeguards and Security Division

U.S. DOE/SF

1333 Broadway

Oakland, CA 94612

1 Warren Jue, Chief

Inspection and Evaluation Branch

U.S. DOE/SF

1333 Broadway

Oakland, CA 94612

1 William G. Phelps, Director

Safeguards and Security Division

U.S. DOE/OR

P.O. BOX 2001

Oak Ridge, TN 37831-8570

1 Branch Chief of Physical Security Safeguards and Security Division U.S. DOE/OR P.O. BOX 2001

Oak Ridge, TN 37831-8570

1 Distribution Services

Office of Scientific and Tech Information

U.S. DOE/OR

P.O. BUX 6\%2

Oak Ridge, TN 37831

1 Robert L. Windus, Security Manager U.S. DOE

Bonneville Power Administration

P.O. BOX 3621

Portland, OR 97208

1 A.C. Walker, Chief Safeguards Branch

U.S. DOE/RL

P.O. BOX 55

Richland, WA 99352

1 Joe W. Wiley, Chief

Operations Security Branch

U.S. DOE/RL

P.O. BOX 55

Richland, WA 99352

1 G. Stefani, Director

Safeguards and Security Division

U.S. DOE

Schenectady Naval Reactors Office

P.O. BOX 1069

Schenectady, NY i2301 
1 Warren C. Sherrand, Jr.

U.S. DOE

Pinellas Area Office

P.O. BOX 2900

Largo, FL 34649

1 J.A. Bullian, Director

Safeguards and Security Division

U.S. DOE/PNR

P.O. BOX 109

West Mifflin, PA 15122

1 Steve Nashatker

Westinghouse Savannah River Co.

703-45A, Rm.150

P.O. BOX 616

Aiken, SC 29802

1 Mark Bolton, Manager

Security Programs \& Projects Dpt

Wackenhut Services, Inc.

P.O. Drawer W

Aiken, SC 29802

1 Wackenhut CTA

Safeguards and Technical

Security Department

P.O. BOX 18041

Albuquerque, NM 87185

1 James Hallihan

Mason \& Hanger-Silas Mason

Pantex Plant

P.O. EOX 30020

Amarillo,Tx 79117

1 Ronald Perry

Argonne National Labs East

Bldg. 222 Electronics

9700 South Cass Avenur:

Argonne, IL 60439

1 R.E. Remley

Rocketdyne Division

Rockwell International Corp.

P.O. BOX 309

Canoga Park, CA 91304

1 James M. Miller, 1... nager Safeguards and Security

Westinghouse Materials Company of Ohio

P.O. BOX 398704

Cincinnati, OH 45239 
1 Terry Hill

Battelle Memorial Institute 505 King Avenue

Columbus, OH 43201-2693

1 Dale Moul

Battelle Memorial Institute 505 King Avenue

Cclumbus, OH 43201-2693

1 Harley Toy, Manager

Nuclear Services

Battelle Memorial Institute 505 King Avenue

Columbus, $\mathrm{OH}$ 43201-2693

1 C.A. Robertson, Division Leader Los Alamos National Laboratory Operational Safeguards and Security P.O. BOX 1663

Los Alamos, NM 87545

1 M.F. Duff

Technologies Department

EG\&G Mound

Bldg. OSE-211

P.O. BOX 300

Miamisburg, OH 45342

3 EG\&G Mound

Attn: K.N. Gardner, M.A. Gibson,

C.L. Fellers

P.O. BOX 300

Miamisburg, OH 45342

1 J. Dollinger,

Security Department

Boeing Petroleum Services

850 South Clearview Parkway

New Orleans, LA 70123

1 Walk Haydell and Associates

600 Carondolet

New Orleans, LA 70130

1 Clifford A. Druit, Manager

Y-12 Safeguards and Security

Martin Marietta Energy Systems

Bldg. 9706-1, MS 8212

P.O. BOX Y

Oak Ridge, TN 37831 
1 Jim Nations

Oak Ridge Gaseous Diffusion

ORGDP Security Department

Bldg. K-1652, MS-351

P.O. BOX P

Oak Ridge, TN 37831

1 A.K. Yancy

Martin Marietta Energy Systems

Paducah Gaseous Diffusion Plant

P.O. BOX 1410

Paducah, KY 42001

1 R.R. Miller

MS 1112

Martin Marietta Energy Systems

P.O. BOX 628

Piketon, $\mathrm{OH} 45661$

1 S.L. Barnes

MS 1232

Martin Marietta Energy Systems

P.O. BOX 628

Piketon, $\mathrm{OH} 45661$

1 Ben G. Essary

West Hanford Company

Security Applications Center

MS L6-09

P.O. BOX 1970

Richland, WA 99352

1 Doug Carlisle, Manager

Safeguards and Security

Pacific Northwest Lab

P.O. BOX 999

Richland, WA 99352

1 Ted Aichele

Pacific Northwest Lab

MS P7-76

P.O. BOX 999

Richland, WA 99352

1 Joe Indusi

Nuclear Engineering Department Brookhaven National Laboratory Bldg. 197C

2400 Upton Road

Upton, NY 11973 
3 L. Runge, G. Schoenen, \& K. Dahms

Brookhaven National Laboratory

Bldg. 50

2400 Upton Road

Upton, NY 11973

1 DCS/Security Police

U.S. Air Forces in Europe APO New York 09012-5001

1 Chief of Security Police AF Systems Command Andrews AFB, :C 20334-5000

1 William A. Wal

FAA Technical Center

ACT-360, Bldg. $\angle 02$

Atlantic City Airport, NJ 08405

1 Defense Intelligence Agency

DIADDX-7B

Bollong AFB, DC 20301-6734

1 A.L. Lavery

Transportation Systems Center Kendall Square

Cambridge, MA 02142

1 Defense Intelligence Agency DIAVOS-2A

Bolling AFB, DC 20301-6734

1 Defense Intelligence Agency DIA/DT-2B

Bolling AFB, DC 20301-6734

1 Chief of Security Police Alaskan Air Command

Elmendorf AFB, AK 95506-5001

1 Belvoir Research, Development and Engineering Center Attn: STRBE-JI (A.Zushin)

Fort Belvoir, VA 22060-5606

1 Belvoir Research, Development and Engineering Center Attn: STRBE-ZM (J.M. Hale) Fort Belvoir, VA 22060-5606 
1 Belvoir Research, Development and Engineering Center

Product Manager

Physical Security Equipment

Attn: AMCPM-PSE

Fort Belvoir, VA 22060-5606

1 Commander

US Army Troop Support Command

Attn: STRBE-1-POLIC (M. Jennings)

Fort Belvoir, VA 22060

1 Commanding General

USAJFKSWCS

SOTIC

Ft. Bragg, NC 28307-5000

1 Commanding General

1st SOCOM

ODCOPS-Special Projects

Ft. Bragg, NC 28307

1 Col. William F. Garrison

Dept. of Army

1st Special Forces Operational

Det.-Delta

Ft. Bragg, NC 28307-5000

1 U.S. Army Military Police School

ATZN-MP-TS (Capt. Sanders)

Ft. McClellan, AL 36205-5030

1 Michael Kraynick

National Security Agency

M51

Ft. Meade, MD 20755

1 Robert D. Dikkers, Leader

Building Security Group

National Bureau of Standards

Gaithersburg, MD 20899

1 485th EIG/EICI

Attn: C. Winters

Griffiss AFB, NY 13441-6348

1 HQ U.S.AF/ESD/AVJ

Hanscom AFB, MA. 01731

1 DCS/Security Police

Pacific Air Forces

Hickam AFB, HI 96853-5001 
1 Commander

U.S. Army Engineering Division Attn: HNDED-ME, Electronic

Technology

P.O. BOX 1600

Huntsville, AL 35806

1 HQ U.S.AF/AFOSP

Attn: Gen. Frank Martin

Kirkland AFB, NM 87117-6001

1 Director of Plans \& Programs (SPP)

Air Force Office of Security Police

Kirkland AFB, NM 87117-5000

1 Director of Operations (SPO)

Air Force Office of Security Police

Kirtland AFB, NM 87117-5000

1 Chief of Security Police

Tatical Air Command

Langley AFB, VA 23665-5000

1 DCS/Security Police

Strategic Air Command

Offutt AFB, NE 68113-5000

1 John Trout

U.S. Army Corps of Engineers, MROED-S

215 North 17th Street

Omaha, NE 68102

1 Chief of Security Police

AF Space Command

Peterson AFB, CO 80914-5001

1 Naval Civil Engineering Laboratory

Attn: G. Cook L-56

Port Hueneme, CA 93043

1 Chief of Security Police

Electronic Security Command

San Antonio, TX 78243-5000

1 Col. Ken Fore, Chairman

U:S. Dept of Defense

DOD Physical Security Action Group

OU.S.DRE/TWP/SP

The Pentagon

Washington, DC 20301 
1 Central Intelligance Agency

Director, Office of Security

202 Jefferson

Washington, DC 20505

1 U.S. Arms Control \& Disarmament Agency

Chief

Nuclear Safeguards \& Tech. Div.

320 21st Street, NW

Washington, DC 20451

1 Raymond Brady, Director

U.S. Nuclear Regulatory Commission

Division of Security

Washington, DC 20555

1 C.C. Slagle, Manager

Technical Division

U.S. Bureau of Engraving \& Printing

Room 303M

14th \& C Street SW

Washington, DC 20228

1 J. Partlow, Director

U.S. Nuclear Regulatory Commission

Division of Inspection Programs

Washington, DC20555

1 Robert Burnett, Director

U.S. Nuclear Regulatory Commission

Division of Safeguards

Mail Stop 881-SSS

Washington, DC 20555

1 Fred Branch, Chief

Physical Security Branch

U.S. Dept. of State

DS/PSD Room 804,SA6

Washington, DC 20520

1 John Lechevet

U.S. Dept. of State

DS/ST/CMP Room 2513

Washington, DC 20520

1 Tom Fey

U.S. Dept. Of State

DS/PI/PRD, State Annex 1

2201 C Street, NW

Washington, DC 20520 
1 Richard J. Solan, Chief

U.S. Secret Service

Security Division/ Planning \&

Development

1800 G Street NW, Rm 941

Washington, DC 20223

1 John C. Hagan

National Aeronautics and Space

Administration

Security Office (NIS)

Washington, DC 20546

1 James W. Atherton, SA

Federal Bureau of Investigation

Washington Field Office

10th St. and Pennsylvania Ave. NW

Washington, DC 20537

1 Stanley W. Zack Jr.

Federal Bureau of Investigation

Washington Field Office

10th St. and Pennsylvania Ave. NW

Washington, DC 20537

1 U.S. Dept. of Justice

Federal Bureau of Prisons

Attn: Jim Mahan, Rm. 300

320 First St. NW

Washington, DC 20534

6612 M. W. Moulton

7433 R. M. Workhoven

7433 P. W. Knight

8401 C. L. Knapp

9249 John Matter

9500 D. S. Miyoshi

9502 R. P. Syler

9502 Video Library

$9503 \mathrm{~J}$. W. Kane

9503 C. L. Schuster

9504 R. W. Moya

9508 R. F. Davis

9521 D. E. McGovern

9531 C. H. Hine

9531 D. J. Gangel

9531 R. W. Madsen

9533 D. C. Hanson

9533 E. R. Sanders

9533 E. D. Niper 


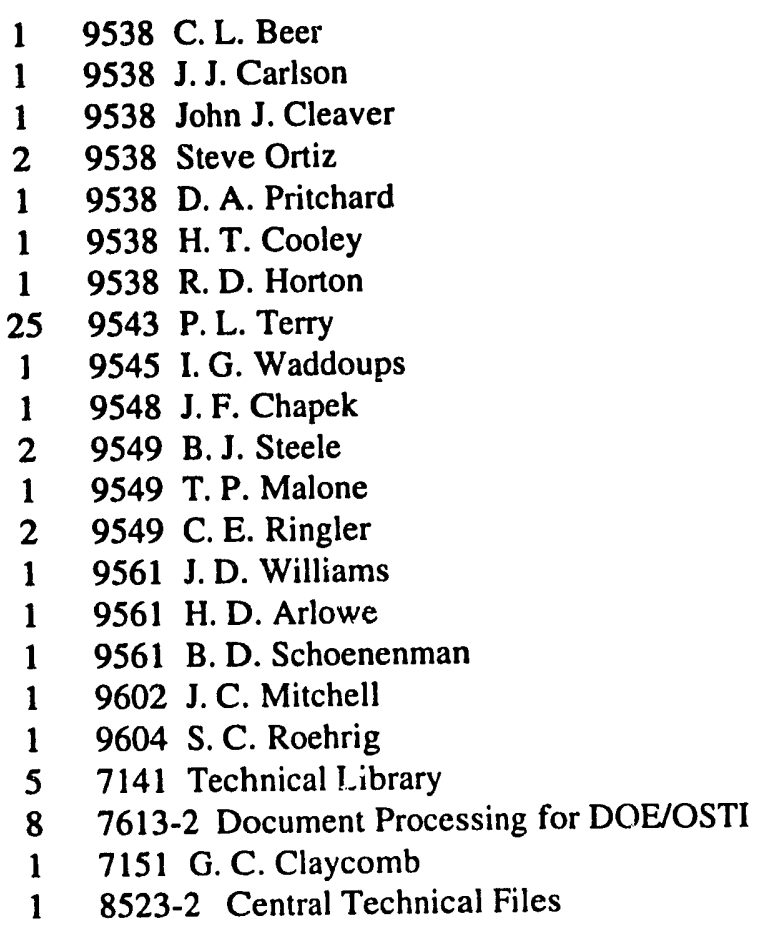



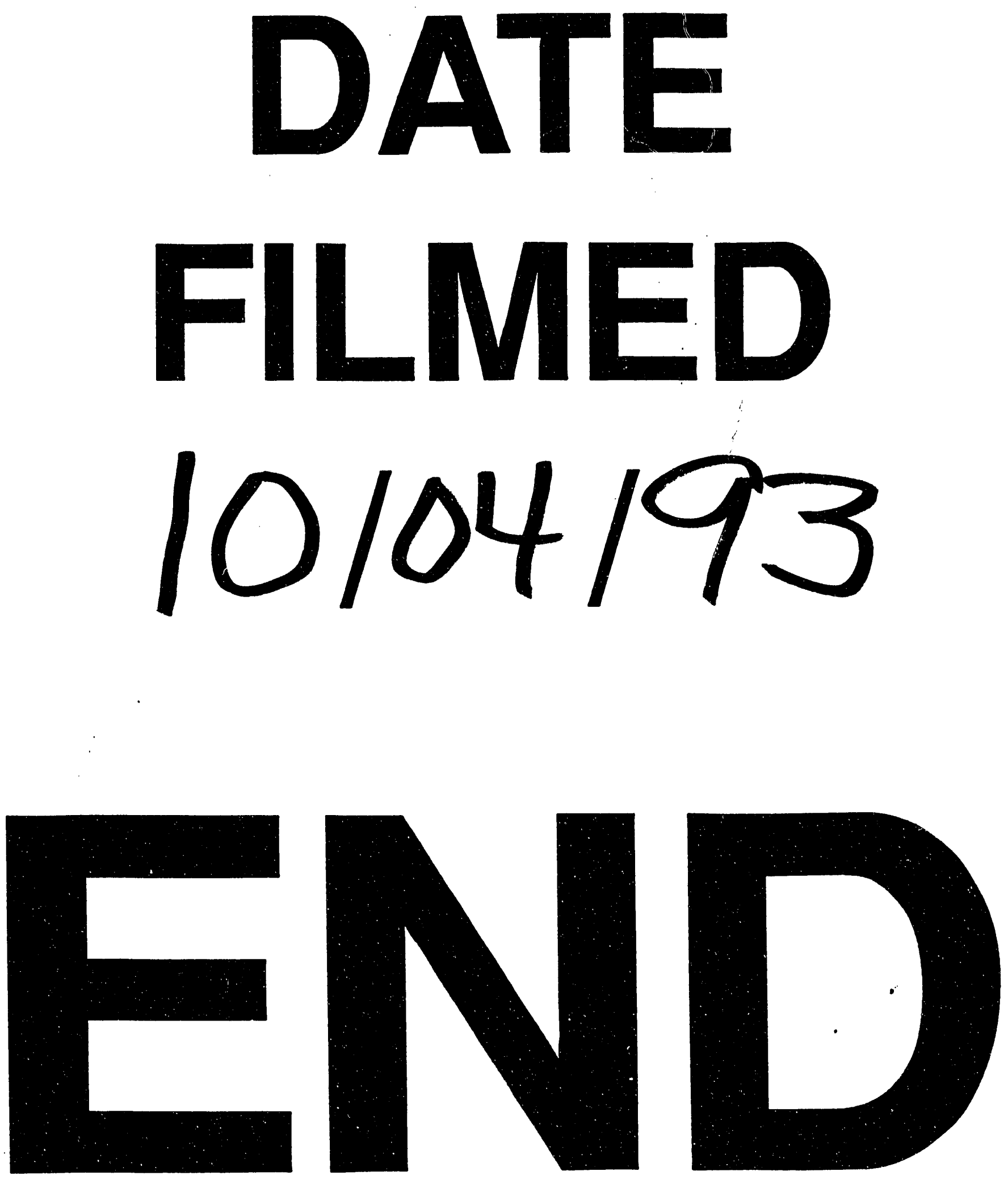
